\title{
Acute Limb Ischemia Due to Retention of Collagen Plug
}

Reema A Sheth ${ }^{1 *}$, Muhammad A Kang ${ }^{1}$, Timothy M Jordan ${ }^{1}$, Nisha A Sheth ${ }^{2}$, and Ibrahim Shah ${ }^{1}$

${ }^{1}$ McLaren Greater Lansing, 4385 Foxpointe, West Bloomfield, MI 48323, USA

${ }^{2}$ American University of Antigua, USA

\begin{abstract}
Vascular closure devices following cardiac catheterization through the femoral artery have been gaining significant traction. Compared to the previous gold standard of manual compression, VCDs allow for shortened time to ambulation, decreased duration of hospitalization post procedure, and quicker hemostasis. Despite the advantages and variety of devices available, there are still complications to take into account. Our patient is a 74-year-old female who presented with intermittent right lower extremity pain. Her symptoms began two weeks prior to presentation, shortly after a recent left heart catheterization that was performed for chest pressure despite normal stress testing. A right lower extremity Doppler demonstrated decreased blood flow and a thrombus was presumed in the superficial femoral artery. After failed attempts of trying to retrieve the thrombus through a spider distal protection device and then a 7-French sheath, the patient was taken to the operating room. Specimens removed were identified as the Angio-Seal plug from the left heart catheterization. In conclusion, risk factors associated with VCD complications should be fully assessed due to the possibility of serious complications. In addition, misinterpretation can lead to delayed or incorrect treatment. Angio-Seal has proven to be highly efficacious, however, further studies will be needed to avoid device related complications.
\end{abstract}

Keywords: Vascular closure device; Collagen plug

\section{Introduction}

Vascular closure devices (VCD) following cardiac catheterization through the femoral artery have been gaining significant traction since their initial use in the mid-1990s [1]. Compared to the previous gold standard of manual compression, VCDs allow for shortened time to ambulation, decreased length of hospitalization post procedure, and quicker hemostasis [2-4]. These devices also display a safety profile comparable to that of manual compression [5]. Despite the many advantages and variety of devices currently available, there are still risks that one must take into account: common complications include device failure, bleeding, and hematoma formation $[1,2,6]$. Less common, albeit more severe, include infection, ischemia, and death $[4,7]$.

\section{Case Report}

Our patient is a 74-year-old Caucasian female with past medical history of hypertension and aortic valve insufficiency who presented to the hospital with a chief complaint of intermittent right lower extremity pain. The patient stated that her symptoms began two weeks prior to presentation, shortly after a recent left heart catheterization that was performed for chest pressure despite normal stress testing.

During her catheterization, the coronary arteries were found to be patient with a left ventricular ejection fraction of approximately $60 \%$ without wall motion abnormalities. For the procedure, the right femoral artery was accessed and a 6-French Angio-Seal VIP vascular closure device was used.

The patient stated that she had been experiencing significant aching pain which encompassed the entirety of her leg after ambulating half a block; rest alleviated the pain. She also reported mild pain in her right groin without accompanying swelling or erythema.

Her symptoms prompted her to follow up with her cardiologist as an outpatient where an arterial Doppler study displayed significant reduction in blood flow in her right lower extremity with a right femoral thrombus. The patient was subsequently directly admitted to the hospital and placed on a heparin infusion. On physical exam, the patient had absent pulses below the right femoral artery; however, no discoloration, change of temperature, sensory, or motor deficits of the extremity were appreciated.

The following morning, the patient underwent angioplasty of the right lower extremity from a contralateral approach. Because of the thrombus burden in the right common femoral artery, a $6 \mathrm{~mm}$ Spider distal protection device was deployed in the right distal superficial femoral artery. Successful angioplasty of the right common femoral artery was performed with a $6 \mathrm{~mm} \times 20 \mathrm{~mm}$ balloon. The stenosis in the right common femoral artery was reduced from $90 \%$ stenosis to less than $30 \%$. After successful angioplasty, the spider distal protection device was attempted to be retrieved via a 0.35 trail blazer catheter, but the spider could not be recovered. Retrieval through a 7-French sheath was attempted but failed. Finally, the sheath with the spider wedged at the tip was attempted, and the sheath came out but the spider was left behind in the left common femoral artery. Due to this failure, the patient was taken to the operating room for vascular surgery where the spider basket was successfully retrieved through the wall of the common femoral artery along with its contents (Figure 1).

Specimens were then sent to pathology, where a foreign body was identified surrounded by soft tissue attached to the spider device (Figure 2). The foreign body was recognized to be the Angio-Seal plug from the left heart catheterization that was previously presumed to be a thrombus.

\section{Discussion}

Promising results have ascertained that Angio-Seal VCDs have

*Corresponding authors: Reema A Sheth, McLaren Greater Lansing, 4385 Foxpointe, West Bloomfield, MI 48323, USA, Tel: 12482290976; E-mail: shethree@gmail.com

Received April 10, 2017; Accepted May 06, 2017; Published May 10, 2017

Citation: Sheth RA, Kang MA, Jordan TM, Sheth NA, Shah I (2017) Acute Limb Ischemia Due to Retention of Collagen Plug. J Cardiovasc Dis Diagn 5: 273. doi: 10.4172/2329-9517.1000273

Copyright: ( 2017 Sheth RA, et al. This is an open-access article distributed under the terms of the Creative Commons Attribution License, which permits unrestricted use, distribution, and reproduction in any medium, provided the original author and source are credited. 


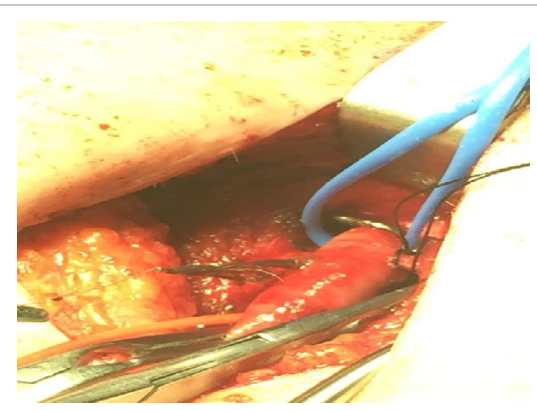

Figure 1: The common femoral artery during the retrieval of the spider basket and presumed thrombus.

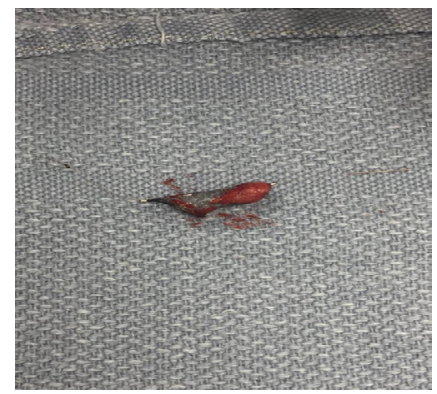

Figure 2: The retrieved "thrombus" attached to a foreign object from the common femoral artery, which was later identified as the Angio-Seal vascular closure device.

excellent efficacy and safety profile after routine catheterization and intervention $[3,5]$. However, clear indications for their use and the risk of complications need to be evaluated and monitored.

Advantages to using a closure device include: faster haemostasis for patients on anticoagulation allowing for immediate anticoagulation post procedure, improved ambulation time, increased patient satisfaction, early discharge, and facilitation of vascular access management of large sheaths $(>10 \mathrm{Fr})[2,7]$. The meta-analysis by Nikolsky et al. evaluated Angio-Seal versus mechanical compression in patients undergoing percutaneous transfemoral coronary procedure. No differences were identified in complication incidence [8]. Another meta-analysis by Chatterjee et al. evaluated whether the use of VCDs are justified; findings displayed that hemostasis was reached much faster in VCDs when compared to compression by an average of 11 minutes, improved time to ambulation by six hours, and reduction of hospital stay length by 7.3 hours. There was also noted to be a $98 \%$ successful deployment rate [9]

VCD complications include hematomas, haemorrhage, retroperitoneal bleed, local discomfort, increased cost, infection, device failure, embolization, pseudoaneurysms, AV fistulas, and vessel thrombosis $[4,7,8,10]$. The meta-analysis by Vaitkus showed a significantly shorter time to haemostasis with VCD usage, increased risk of infection $(0.6 \%$ vs $0.2 \%, \mathrm{P}=0.02)$, lower limb ischemia, arterial stenosis, device entrapment $(0.3 \%$ vs $0 \%, \mathrm{P}=0.07)$, and need for vascular surgery $(0.7 \%$ vs $0.4 \%, \mathrm{P}=0.10)[4]$.

Despite clear advantages related to the use of VCDs, there are variables that can affect the risk for complications; artery size and anticoagulated status being two such risk factors [1]. If a patient is undergoing cardiac catheterization with a small sheath and minimal bleeding risk, manual compression may be sufficient. However, if the patient just underwent percutaneous coronary intervention and immediate anticoagulation is needed, the benefits of a closure device may out-weigh the associated risks [1]. VCD contraindications include small femoral arteries $(<5 \mathrm{~mm})$, severe atherosclerosis or calcification of the common femoral artery and high or low common femoral stick [7].

Studies have also indicated that distinctive VCDs have unique complication risks [2] and Angio-Seal has been found to be highly efficacious in comparison to other VCDs $[2,8,11]$. A prospective study by Applegate et al. evaluated 1,004 patients where an Angio-Seal VCD was utilized and found a $99.7 \%$ success rate of deployment; this was independent of prior experience indicating a minuscule learning curve [3]

The meta-analysis studies by Nikolsky et al. and Koreny et al. acknowledged that despite VCDs being efficacious, there may be heterogeneity among current studies used for data with different endpoints [8,10]. Additional studies need to focus on patient satisfaction, newer generation VCDs, and improved user expertise [11]. Furthermore, studies will need to focus on transradial versus transfemoral approaches [12] and how these methods will affect current risks and benefits.

\section{Conclusion}

Risk factors associated with VCD complications should be fully assessed due to the possibility of serious complications. In addition, misinterpretation can lead to delayed or incorrect treatment. In our patient, catheter-assisted removal of a presumed thrombus was performed. Surgical exploration should be considered as a high priority for the risk of collagen plug embolization after the use of VCDs [7]. Angio-Seal has proven to be highly efficacious, however, further studies will be needed to avoid device related complications [11]

\section{References}

1. Applegate R (2012) Vascular closure device failure. JACC: Cardiovascular Interventions 5: 845-847.

2. Vidi VD, Matheny ME, Govindarajulu US, Normand ST, Robbins SL, et al (2012) Vascular closure device failure in contemporary practice. JACC: Cardiovascular Interventions 5: 837-844.

3. Applegate R, Turi Z, Sachdev N (2010) The angio-seal evolution registry: Outcomes of a novel automated angio-seal vascular closure device. J Invasive Cardiol 22: 420-426

4. Vaitkus P (2004) A meta-analysis of percutaneous vascular closure devices after diagnostic catheterization and percutaneous coronary intervention. J Invasive Cardiol 16: 243-246.

5. Arora N, Matheny M, Sepke C, Resnic F (2007) A propensity analysis of the risk of vascular complications after cardiac catheterization procedures with the use of vascular closure devices. Am Heart J 153: 606-611.

6. Warren B, Warren S, Miller S (1999) Predictors of complications and learning curve using the Angio-Seal closure device following interventional and diagnostic catheterization. Catheter Cardiovasc Interv 48: 162-166.

7. Nanjundappa A, Jain A (2012) Closure device complications. Complications of interventional cardiovascular procedures: A case-based atlas, demos medical publishing, NY, USA, 23-26.

8. Nikolsky E, Mehran R, Halkin A, Aymong ED, Mintz GS, et al. (2004) Vascular complications associated with arteriotomy closure devices in patients undergoing percutaneous coronary procedures: A meta-analysis. J Am Coll Cardiol 44: 1200-1209.

9. Chatterjee S, Biondi-Zoccai G, Mukherjee D (2013) Vascular closure devices: Are their use justified? evidence from a meta-analysis of randomized trials JACC 61: E1681.

10. Koreny M, Riedmuller E, Nikfardjam M, Siostrzonek P, Müllner M (2004) Arteria puncture closing devices compared with standard manual compression after cardiac catheterization: Systematic review and meta-analysis. JAMA 291: 350-357.

11. Schwartz BG, Burstein S, Economides C, Kloner RA, Shavelle DM, et al. (2010) Review of vascular closure devices. J invasive cardiol 22: 599-607.

12. Baker N, Lipinski M, Escarcega R, Magalhaes MA, Minha S, et al. (2014) TCT844 active versus passive anchoring vascular closure devices: A safety and efficacy comparative analysis. JACC 64: B246. 